\title{
Preparation and Evaluation of Micro particulate Drug Delivery Systems of Gliclazide Employing Starch Acetate
}

\author{
P Veera Lakshmi ${ }^{1}$, KPR Chowdary ${ }^{2 *}$, A Prameela Rani ${ }^{3}$ and VUM Prasad ${ }^{4}$ \\ ${ }^{1}$ PhD Scholar, School of Pharmacy, JNTUK, India \\ ${ }^{2}$ Research Director, Vikas Institute of Pharmaceutical Sciences, India \\ ${ }^{3}$ University College of Pharmaceutical Sciences, ANU, India \\ ${ }^{4}$ Programme Director, School of Pharmacy, JNTUK, India
}

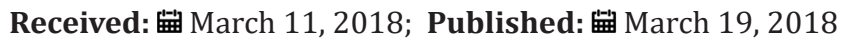

*Corresponding author: KPR Chowdary, Research Director, Vikas Institute of Pharmaceutical Sciences, Rajahmundry, India

\begin{abstract}
Recently much emphasis is being laid on the development of micro particulate DDS in preference to single unit systems because of their potential benefits such as increased bioavailability, reduced risk of systemic toxicity, reduced risk of local irritation and predictable gastric emptying. The objective of the present study is to prepare and evaluate micro particulate drug delivery systems of Gliclazide using starch acetate, a new modified starch for oral controlled release. The starch acetate (DS 2.75) was freely soluble in chloroform and insoluble in several aqueous fluids and organic solvents. Chloroform could be used as solvent for starch acetate in the preparation of micro particles, microcapsules and in film coating Spherical starch acetate- Gliclazide micro particles could be prepared by the emulsification-solvent evaporation method. The method is industrially feasible as it involves emulsification and removal of the solvent, which can be controlled precisely. The emulsification solvent evaporation method was reproducible with regard to size and size distribution of the micro particles. About $65-70 \%$ of micro particles in each batch were in the size range $35 / 50$ mesh $(398.5 \mu \mathrm{m})$ Encapsulation efficiency was in the range 96.0-99.3 \% in the preparation of micro particles.

Gliclazide release from the starch acetate micro particles was slow and spread over longer periods of time. The drug release depended on the proportion of core: coat in the micro particles. A good linear relationship $\left(\mathrm{R}^{2}=0.826\right)$ between percent coat and release rate $\left(\mathrm{k}_{\mathrm{o}}\right)$ was observed. The relationship could be expressed by the linear equation, $\mathrm{y}=12.18-0.173 \mathrm{x}$ where $\mathrm{x}$ is percent coat and $y$ is release rate $\left(\mathrm{k}_{\mathrm{o}}\right)$. Gliclazide release from the starch acetate micro particles was by non fickian (anomalous) diffusion. Formulation F2 prepared using a Core: coat ratio of 8:2 gave slow, controlled and complete release (100\%) of Gliclazide over 12 hours. As such formulation F2 is considered as a promising micro particulate DDS for oral control release of Gliclazide over 12 hours for b.i.d administration
\end{abstract}

Keywords: Multi particulate drug delivery systems; Starch acetate; Gliclazide; Oral controlled release

\section{Introduction}

The design of micro particulate drug delivery systems is an efficient technique to provide the sustained \& controlled delivery of drugs over long periods of time. Micro particulate drug delivery systems [1] consist of small particles of solids or small droplets of liquids surrounded by walls of natural \& synthetic polymer films of varying thickness $\&$ degree of permeability acting as a release rate controlling substance $\&$ have a diameter up to the range of $0.1 \mu \mathrm{m}$ $200 \mu \mathrm{m}$. Micro particulate dosage forms [2] are pharmaceutical formulations in which the active substance is present as a number of small independent subunits. To deliver the recommended total dose, these subunits are filled into capsules, encapsulated or compressed into a tablet. Micro particulate drug delivery systems contain discrete particles that make up a multiple-unit system. They provide many advantages over single-unit systems because of their small size. Multi particulates are less dependent on gastric empty time, resulting in less inter and intra-subject variability in 
gastrointestinal transit time. They are also better distributed and less likely to cause local irritation [3]. Recently much emphasis is being laid on the development of micro particulate dosage forms in preference to single unit systems because of their potential benefits such as increased bioavailability, reduced risk of systemic toxicity, reduced risk of local irritation and predictable gastric emptying [4].

Design of micro particulate drug delivery systems requires a suitable polymer to serve the intended purpose. Several polymers such as benzyl cellulose, cellulose nitrate, cellulose acetate, epoxy resin, ethyl cellulose, polyethylene, polymethyl methacrylate, polystyrene, polyvinyl acetate, Eudragit S-100, chitosan have been used in the design of micro particulate drug delivery systems $[5,6]$. In the present investigation Starch acetate, a new modified starch was tried for the preparation of micro particulate drug delivery systems of Gliclazide for oral controlled release. Starch acetate is reported $[7,8]$ to have excellent bond forming ability and suitable for coating and controlled release applications. The objective of the present study is to prepare and evaluate micro particulate drug delivery systems of Gliclazide using starch acetate for oral controlled release. Gliclazide is a potential second generation, short-acting sulfonylurea oral hypoglycemic agent widely used for the treatment of non-insulin-dependent diabetes mellitus [9]. In general, rapid gastrointestinal absorption is required for oral hypoglycemic drugs in order to prevent a sudden increase in blood glucose level after food intake in patients with diabetes mellitus. However, the absorption rate of Gliclazide from the gastrointestinal tract is slow and varied among subjects. Slow absorption of a drug usually originates from either poor dissolution of the drug from the formulation or poor permeability of the drug across the gastrointestinal membrane [10] .The dose of Gliclazide is $40-80 \mathrm{mg}$ as conventional tablets and $60 \mathrm{mg}$ as sustained release tablets. The conventional tablets are to be taken 2-3 times a day to maintain normal plasma glucose levels. Sustained release formulations offer better patient complains by reducing the frequency of dosage administrations and also provide continuous effect. Emulsificationsolvent evaporation method was tried for the preparation of starch acetate-Gliclazide micro particles.

\section{Materials and Methods}

\section{Materials}

Gliclazide was a gift sample from M/s Micro Labs Limited, Starch acetate (DS 2.75) was obtained from M/s Esai Pharma technology Pvt. Ltd. Potato starch (SD Fine chemicals), and acetic anhydride (Qualigens), sodium hydroxide (Qualigens) and chloroform (Qualigens) were procured from commercial sources. All other materials used were of pharmacopoeia grade.

\section{Methods}

Estimation of Gliclazide: An UV Spectrophotometric method based on the measurement of absorbance at $227 \mathrm{~nm}$ in phosphate buffer of $\mathrm{pH} 7.4$ was used for the estimation of Gliclazide. The method was validated for linearity, accuracy, precision and interference by the excipients. The method obeyed Beer's law in the concentration range of $1-10 \mu \mathrm{g} / \mathrm{ml}$. When a standard drug solution was repeatedly assayed $(n=6)$, the relative error and coefficient of variance (RSD) were found to be $0.80 \%$ and $1.2 \%$ respectively. No interference by the excipients used in the study was observed.

Preparation of Starch Acetate-Gliclazide Micro Particulate DDS: An emulsification solvent evaporation method was tried for preparation of starch acetate-Gliclazide micro particulate DDS. Starch acetate $(0.2 \mathrm{~g})$ was dissolved in chloroform $(10 \mathrm{~mL})$ to form a homogeneous solution. Core material, Gliclazide $(0.8 \mathrm{~g})$ was added to the polymer (starch acetate) solution $(5 \mathrm{ml})$ and mixed thoroughly. The resulting mixture was then added in a thin stream to $200 \mathrm{ml}$ of an aqueous mucilage of sodium CMC $(0.5 \% \mathrm{w} / \mathrm{v})$ contained in a $450 \mathrm{ml}$ beaker while stirring at $1000 \mathrm{rpm}$ to emulsify the added dispersion as fine droplets. A Remi medium duty stirrer with speed meter (model RQT 124) was used for stirring. The solvent, chloroform was then removed by continuous stirring at room temperature $\left(28{ }^{\circ} \mathrm{C}\right)$ for $3 \mathrm{~h}$ to produce spherical micro particles. The micro particles were collected by vacuum filtration and washed repeatedly with water. The product was then air dried to obtain discrete micro particles. Different proportions of core: coat namely 9:1 (F1), 8:2 (F2), 7:3 (F3) and 6:4(F4) were used to prepare micro particles with varying amount of coat polymer.

Estimation of Drug Content and Encapsulation Efficiency: Four samples of $100 \mathrm{mg}$ each were taken from each batch of micro particles prepared and assayed for Gliclazide content at $227 \mathrm{~nm}$ .Encapsulation efficiency was estimated using the equation,

Encapsulation efficiency (\%)=[Estimated drug content, \%/ Theoretical drug content\%] X100

Size Analysis: For the size distribution analysis, different fractions in a batch were separated by sieving using a range of standard sieves. The amounts retained on different sieves were weighed.

Drug Release Study: Release of Gliclazide from the micro particles of size 20/30 and 30/50 mesh was studied in phosphate buffer of pH $7.4(900 \mathrm{ml})$ using an 8 station dissolution rate test apparatus (model Disso-2000, M/s Lab. India) with a paddle stirrer( Apparatus 2) at $50 \mathrm{rpm}$. A temperature of $37^{\circ} \pm 1^{\circ} \mathrm{C}$ was maintained throughout the experiment. A sample of micro particles equivalent to $60 \mathrm{mg}$ of Gliclazide was used in each test. Samples (5 ml) were withdrawn through a filter $(0.45 \mu)$ at different time intervals over $12 \mathrm{~h}$ and were assayed at $227 \mathrm{~nm}$ for Gliclazide content. The sample ( $5 \mathrm{ml}$ ) taken at each sampling time was replaced with drug free dissolution fluid and a suitable correction was applied for the amount of drug lost in sampling for the estimation of amount of drug released at various times. Each drug release experiment was conducted in triplicate $(\mathrm{n}=3)$. 
Analysis of Release Data: Drug release data were analyzed as per zero order, first order, Higuchi [11] equation and KorsmeyerPeppas [12] equation models to assess the release kinetics and mechanism.

\section{Results and Discussion}

The starch acetate (DS 2.75) was insoluble in water, aqueous buffers of $\mathrm{pH} 1.2$ and 7.4, methanol, petroleum ether, dichloromethane and cyclohexane. It is freely soluble in chloroform. Hence chloroform was used as the solvent for starch acetate in the preparation of micro particulate DDS. An emulsification - solvent evaporation method was used for the preparation of micro particles of starch acetate-Gliclazide. The method involves emulsification of the polymer (starch acetate) solution in chloroform containing the dispersed drug particles in an immiscible liquid medium $(0.5 \% \mathrm{w} / \mathrm{v}$ solution of sodium CMC) as micro droplets, followed by removal of the solvent, chloroform by continuous stirring to form rigid micro particles. The micro particles were collected by vacuum filtration and washed repeatedly with water. The product was then air dried to obtain discrete micro particles. The micro particles were found to be discrete, spherical and free flowing. The sizes could be separated readily by sieving and a more uniform size range of micro particles could easily be obtained. The sieve analysis of different batches of micro particles prepared indicated that a large proportion,65-70\%, in each batch were in the size range of $35 / 50$ mesh $(398.5 \mu \mathrm{m})$. The reproducibility of the method with regard to size distribution of the micro particles was evaluated by preparing three batches of micro particles under identical conditions in each case. Size analysis indicated that about $65-70 \%$ of the micro particles are in the size range 35/50 mesh in all the batches. Microparticles of the size $(398.5 \mu \mathrm{m})$ were selected for further evaluation.

The physical characteristics of the micro particulate DDS prepared are given in Table 1. Low coefficient of variation (cv) in percent drug content $(<2.0 \%)$ indicated uniformity of drug content in each batch of micro particles. The encapsulation efficiency was in the range 96.0-99.3\%. Drug content of the micro particles was found to be the same in the two sizes, 20/35, 35/50 mesh. At-test of significance indicated that the difference in the drug content of the two sizes in each case is not significant $(\mathrm{P}>0.05)$. Table 1 Gliclazide release from the various micro particles of size $35 / 50$ was studied in phosphate buffer $\mathrm{pH}$ 7.4. The drug release profiles are shown in Figure1. The release data were analyzed as per Zero order, First order, Higuchi [11] equation and Korsmeyer-Peppas [12] equation models to assess the release kinetics and mechanism. The kinetic parameters ( $\mathrm{r} 2$ values, rate constants and $\mathrm{n}$ values) in the analysis of release data as per various kinetic models are given in (Table 2).

Table 1: Physical Characterstics of the Microparticulate DDS Prepared.

\begin{tabular}{|c|c|c|c|c|c|c|}
\hline DDS & Mesh Size & Mean size $(\boldsymbol{\mu m})$ & Core:Coat ratio & Gliclazide content(\%) $\overline{\mathbf{x}} \pm \mathbf{s d})$ & Encapsu lation efficiency(\%) & Percent Coat Polymer \\
\hline \multirow{2}{*}{ F1 } & $20 / 35$ & 670 & $9: 1$ & $87.2 \pm 1.6$ & 96.9 & 12.8 \\
\cline { 2 - 7 } & $35 / 50$ & 398.5 & $9: 1$ & $86.4 \pm 1.4$ & 96.0 & 13.6 \\
\hline \multirow{2}{*}{ F2 } & $20 / 35$ & 670 & $8: 2$ & $79.2 \pm 1.2$ & 993 & 20.8 \\
\cline { 2 - 7 } & $35 / 50$ & 398.5 & $8: 2$ & $78.6 \pm 1.4$ & 98.3 & 21.4 \\
\hline \multirow{2}{*}{ F3 } & $20 / 35$ & 670 & $7: 3$ & $69.5 \pm 1.3$ & 99.3 & 30.5 \\
\cline { 2 - 7 } & $35 / 50$ & 398.5 & $7: 3$ & $68.4 \pm 1.4$ & 97.7 & 31.6 \\
\hline \multirow{2}{*}{ F4 } & $20 / 35$ & 670 & $6: 4$ & $58.2 \pm 1.2$ & 97.0 & 41.8 \\
\cline { 2 - 7 } & $35 / 50$ & 398.5 & $6: 4$ & $58.4 \pm 1.1$ & 97.3 & 41.6 \\
\hline
\end{tabular}

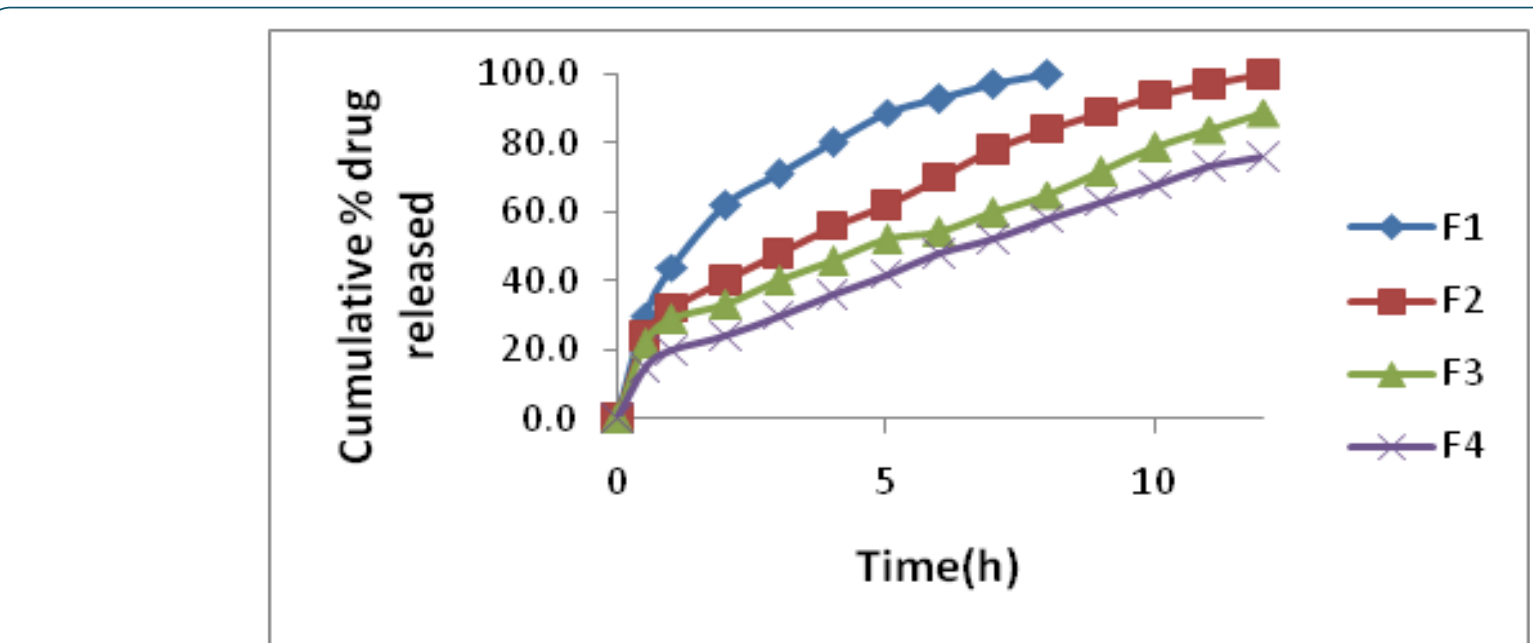

Figure 1: Gliclazide Release Profiles of Various Micro particulate DDS Prepared 
Gliclazide release from all the starch acetate micro particles tested was slow and spread over longer periods of time. The release depended on proportion of core and coat in the micro particles. As the coat proportion was increased the release rate was decreased. A good linear relationship $\left(\mathrm{R}^{2}=0.826\right)$ between percent coat and release rate $\left(\mathrm{k}_{0}\right)$ was observed as shown in Figure 2. The relationship could be expressed by the linear equation, $\mathrm{y}=12.18-0.173 \mathrm{x}$ where $\mathrm{x}$ is percent coat and $y$ is release rate $\left(\mathrm{K}_{\mathrm{o}}\right)$. A comparison of $\mathrm{R}^{2}$ values in various models revealed that the $\mathrm{R}^{2}$ value was higher in the case of korsmeyer peppas model in all the cases. As such the release data of all the micro particles tested obeyed korsmeyer peppas equation model which indicates that the drug release from the micro particles was by diffusion mechanism. The release exponent Table 2: Kinetic Parameters (R2 Values, Rate Constants and n values) in the Analysis of Release Data as per Various Kinetic Models.

\begin{tabular}{|c|c|c|c|c|c|c|c|}
\hline \multirow{2}{*}{ DDS } & \multicolumn{2}{|c|}{ Zero order } & \multicolumn{2}{|c|}{ First order } & \multirow{2}{*}{$\begin{array}{c}\text { Higuchi } \\
\text { R2 }\end{array}$} & \multicolumn{2}{|c|}{ Korsemeyer Peppas } \\
\hline & KO & $\mathbf{R 2}$ & R2 & K1 & & $\mathbf{n}$ & $\mathbf{R 2}$ \\
\hline $\mathrm{F} 1$ & 10.8 & 0.8502 & 0.9800 & 0.4604 & 0.9898 & 0.454 & 0.9950 \\
\hline $\mathrm{F} 2$ & 7.4 & 0.9397 & 0.9300 & 0.3220 & 0.9663 & 0.471 & 0.9979 \\
\hline F3 & 6.2 & 0.9522 & 0.9410 & 0.1612 & 0.9195 & 0.454 & 0.9886 \\
\hline F4 & 5.6 & 0.9765 & 0.9853 & 0.1150 & 0.9317 & 0.542 & 0.9965 \\
\hline
\end{tabular}

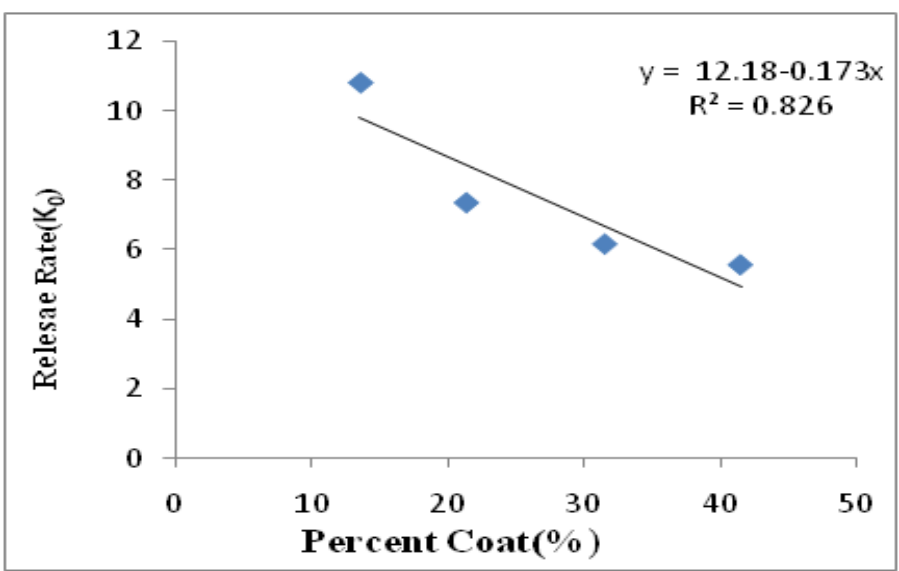

Figure 2: Relationship between Percent Coat and Release Rate $\left(\mathrm{K}_{0}\right)$ of Micro particulate DDS (Size 398.5 $\left.\mu \mathrm{m}\right)$

\section{Conclusion}

a) The starch acetate (DS 2.75) was freely soluble in chloroform and insoluble in several aqueous fluids and organic solvents.

b) Chloroform could be used as solvent for starch acetate in the preparation of micro particles, microcapsules and in film coating.

c) Spherical starch acetate- Gliclazide micro particles could be prepared by the emulsification-solvent evaporation method. The method is industrially feasible as it involves emulsification and removal of the solvent, which can be controlled precisely.

d) The emulsification solvent evaporation method was reproducible with regard to size and size distribution of the micro particles. About $65-70 \%$ of micro particles in each batch were in the size range $35 / 50$ mesh $(398.5 \mu \mathrm{m})$. (n) in korsmeyer peppas equation model was in the range 0.454 0.542 in all the cases indicating that the drug release from the micro particles was by non-fickian (anomalous) diffusion (Table 2). The results of the present study, thus, indicated that starch acetateGliclazide micro particles could be prepared by emulsification solvent evaporation method using chloroform as solvent for starch acetate. These micro particles could be used for oral control release of Gliclazide. Formulation F2 prepared using a Core: coat ratio of 8:2 gave slow, controlled and complete release (100\%) of Gliclazide over 12 hours. As such formulation F2 is considered as a promising micro particulate DDS for oral control release of Gliclazide over 12 hours for b.i.d administration. 
as a promising micro particulate DDS for oral control release of Gliclazide over 12 hours for b.i.d administration.

\section{References}

1. Lwise Donald (2000) Handbook of Pharmaceutical Controlled Release Technology. New York, USA.

2. Sharma S, Pawar A (2006) Low Density Multi particulate System for Pulsatile Release of Meloxicam. Int J Pharm 313(1-2): 150-158.

3. Laila FA, Chandran S (2006) Multiparticulate Formulation Approach to Colon Specific Drug Delivery Current Perspectives. J Pharm Pharm Sci $9(3): 327-338$

4. (2002) Preparing Modified Release Multiparticulate Dosage Forms with Eudragit Polymers. Pharma Polymers 9: 2-3.

5. Satheesh Madhav NV, Shivani Kala (2011) Review on Microparticulate Drug Delivery System. International Journal of Pharm Tech Research 3(3): 1242-1254

6. Sujata Kumari, Meenu Nagpal ,Geeta Aggarwal, Puneet, Upendra K Jain, et al. (2016) Microparticles Drug Delivery System. A Review World Journal of Pharmacy and Pharmaceutical Sciences 5(03): 543-566.
7. Tarvainen M, Sumwen R, Peltonen S, Tiihonen P, Paroneni P (2002) Starch acetate--a novel film-forming polymer for pharmaceutical coatings. J Pharm Sci 91(1): 282-289.

8. Korhonen O, Raatikainen P, Harjunen P, Nakari J, Suihko E, et al. (2000) Starch acetates--multifunctional direct compression excipients. Pharm Res 17: 1138 .

9. Schernthaner G (2003) Gliclazide Modified Release: A Critical Review Of Pharmacodynamic, Metabolic, And Vasoprotective Effects. Metabolism 52(8 suppl): 29-34.

10. Campbell DB, Lavielle R, Nathan C (1991) the Mode of Action and Clinical Pharmacology of Gliclazide: A Review. Diabetes Res Clin Pract 14: S21-S36.

11. Higuichi T (1963) Mechanism of Sustained-action medication. Theoretical analysis of rate of release of solid drugs dispersed in solid matrices. J Pharm Sci 52(12): 1145-1149.

12. Korsemeyer RW, Gurny R, Doelkar E, Buri P, Peppas NA (1983) Mechanism of Solute Release from Porous Hydrophilic Polymers. Int J Pharm 15(1): 25-35.

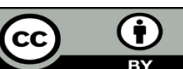

This work is licensed under Creative Commons Attribution 4.0 License

Submission Link:

Submit Article

DOI: 10.32474/DDIPIJ.2018.01.000103

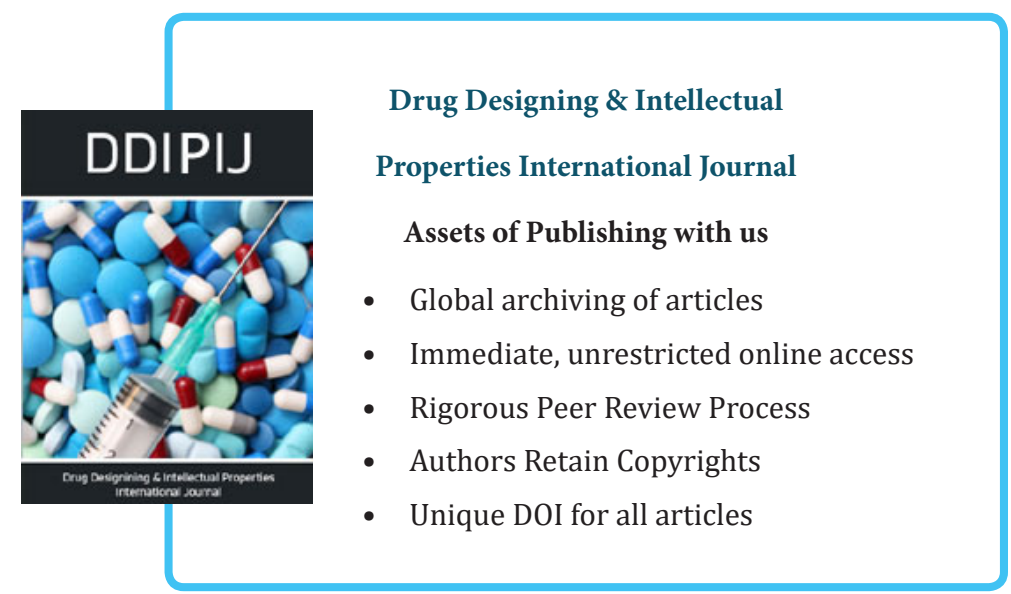

